\title{
Tumores malignos de ovario en niñas y adolescentes
}

\author{
Drs. Jorge E. Zúñiga * - E. Iván Ortiz**
}

Los tumores de ovario en niñas y adolescentes son raros y comprenden en promedio, $1 \%$ de todas las neoplasias malignas entre los 0-17 años $(1,2,3,5)$.

La frecuencia de tumores en el ovario varía con la edad de la paciente y la clasificación histológica de la neoplasia (5).

La rareza de los tumores ováricos en niñas y la dificultad en obtener una recopilación estadística significativa han insidido en la falta de publicaciones científicas en la literatura médica.

El presente estudio tiene como objetivo el análisis del comportamiento de esta patología en la ciudad de Cali, Col. S.A. durante un período de tiempo de 22 años.

\section{PACIENTES Y METODOS}

Se hizo una revisión de las historias clínicas de las pacientes entre 1 y 18 años de

\footnotetext{
* Profesor Asociado Univalle Jefe Servicio de Ginecología Hospital Universitario Cali

** Profesor Auxiliar Univalle Hospital Universitario Cali
}

edad, que tuvieron tumores malignos de ovario confirmados por estudio histológico entre los años de 1962 y 1984.

Todas las pacientes provienen de la ciudad de Cali y su identificación se hizo mediante la revisión del archivo del Registro de Cáncer del Departamento de Patología de la Universidad del Valle.

Las variables analizadas para cada caso fueron:

Edad

Síntomas que indujeron el diagnóstico

Tipo histológico del tumor

Estadio clínico

Tratamiento realizado

Recuperación, evaluada por el seguimiento de cada caso por lo menos durante 1 año; y en 5 casos hasta la muerte.

\section{RESULTADOS}

El promedio de tumores malignos de ovario en la mujer adulta en nuestro medio es de 15 casos por año, cuando el promedio de casos en niñas y adolescentes es de un caso por año.

En el cuadro 1 se puede ver la distribución por grupos de edad. 
TUMORES MALIGNOS DE OVARIO EN NIÑAS Y ADOLESCENTES

\section{VARIABLES POR CASO}

- Edad

- Síntomas

- Tipo histológico

- Estadio clínico

- Tratamiento

- Recuperación

CUADRO No. 1

\begin{tabular}{|c|c|}
\hline EDAD & CASOS \\
\hline $1-4$ & 0 \\
$5-9$ & 4 \\
$10-14$ & 5 \\
$14-18$ & 9 \\
\hline TOTAL & 18 \\
\hline
\end{tabular}

CUADRO No. 2

\begin{tabular}{|l|c|c|}
\hline SINTOMAS & CASOS & $\%$ \\
\hline Masa abdominal & 9 & 50.0 \\
Dolor & 5 & 27.7 \\
Pérdicla de peso & 4 & 22.2 \\
Fiebre & 3 & 16.6 \\
A scitis & 1 & 5.5 \\
\hline
\end{tabular}

Se puede observar mayor incidencia de esta patología en el grupo de $14-18$ años de edad. En el presente estudio no se presentaron casos por debajo de los 7 años de edad.
La manifestación clínica que llevó a la sospecha diagnóstica de tumor de ovario en la mayoría de los casos fue la presencia de masa palpable abdominal en el $90 \%$ de los casos, seguido de dolor y pérdida de peso. La ascitis estuvo presente en el10\% de los casos (ver cuadro 2).

\section{TIPO HISTOLOGICO}

Los turnores más frecuentes en todos los grupos de edad fueron los originados de las células germinales, el tipo histológico más común fue el disgerminoma, al avanzar la edad aumentan en frecuencia los tumores del estroma gonadal y los de origen epitelial, en especial después de la menarca. Después de la pubertad el equilibrio sigue desviándose hacia un promedio de lesiones del tipo seroso y mucinoso papilar que son los más frecuentes encontrados en el adulto. (Ver cuadro 3 ).

\section{CUADRO No. 3}

\begin{tabular}{|c|c|c|c|c|c|}
\hline \multirow[b]{2}{*}{ TIPO HISTOLOGICO } & \multicolumn{3}{|c|}{ GRUPOS DE EDAD } & \multirow[b]{2}{*}{ TOTAL } & \multirow[b]{2}{*}{$\%$} \\
\hline & $5-9$ & $10-14$ & $14-18$ & & \\
\hline 1. Disgerminoma & 1 & 3 & 4 & 8 & 44.4 \\
\hline 2. T.S. Endodérmicos & 2 & 1 & 1 & 4 & 22.2 \\
\hline 3. T.C. de la Granulosa & 1 & - & 1 & 2 & 11.1 \\
\hline $\begin{array}{l}\text { 4. Cistoadenocarcinoma } \\
\text { Seroso }\end{array}$ & - & - & 2 & 2 & 11.1 \\
\hline 5. Cistoadenocarcinoma & & & & & \\
\hline $\begin{array}{l}\text { Mucinoso } \\
\text {. }\end{array}$ & $\overline{-}$ & ; & 1 & 1 & 5.1 \\
\hline 6. Adenocarcinoma & - & 1 & - & 1 & 5.1 \\
\hline TOTAL & & & & 18 & \\
\hline
\end{tabular}

La gran mayoría de las pacientes al momento del diagnóstico, la enfermedad estaba en el Estadio Clínico III.

\section{DISCUSION}

En términos generales los tumores malignos en niñas y adolescentes se tratan igual que en la mujer adulta. Puede ser la excepción los tumores estadios I, los cuales pueden tratarse con cirugía conservadora, quiere esto decir salpingo-ooforectomía unilateral. 
En el cuadro No. 4 se describe el tipo histológico del tumor, su estadio clínico, el tratamiento aplicado y la recuperación posterior de la paciente.

Si los tumores corresponden a estados más avanzados, la radioterapia y la quimioterapia juega un papel muy importante en la recuperación de las pacientes.
Llama la atención en este estudio la gran mortalidad asociada a los casos de disgerminoma, teniendo en cuenta que es un tumor muy radiosensible y que en la mayoría de los casos se utilizó la radioterapia como tratamiento, este resultado puede estar influenciado por problemas de técnica y equipos utilizados en la década del 60.

CUADRO No. 4

\begin{tabular}{|c|c|c|c|c|}
\hline CASO & TIPO HISTOLOGICO & ESTADO & TRAT'AMIENTO & RECUPERACION \\
\hline 1 & Senos Endodérmicos & III & SOD Radioterapia & 5 meses \\
\hline 2 & Senos Endodermicos & I A & SOD & 8 años Neg. \\
\hline 3 & Senos Endodérmicos & III & $\begin{array}{l}\text { Anexohist } \\
\text { Radioterapia y } \\
\text { Quimioterapia }\end{array}$ & $\begin{array}{l}\text { A los } 13 \text { meses } \\
\text { Murio. }\end{array}$ \\
\hline 4 & Senos Endodérmicos & III & Radioterapia & 2 Meses Neg. \\
\hline 5 & Disgerminoma & I A & $5.0 . i$ & 9 años Neg. \\
\hline 6 & Disgerminoma & III & $\begin{array}{l}\text { Anexo Histerect } \\
\text { Radioterapia }\end{array}$ & $\begin{array}{l}\text { Muris a los } 2 \\
\text { meses. }\end{array}$ \\
\hline 7 & Disgerminoma & III & Radioterapia & $\begin{array}{l}\text { Murio a los } 15 \\
\text { Meses. }\end{array}$ \\
\hline 8 & Disgerminoma & III & Radioterapia & Murio al mes. \\
\hline 9 & Disgerminoma & III & $\begin{array}{l}\text { Anexohist. } \\
\text { Radioterapia }\end{array}$ & $\begin{array}{l}\text { A los } 5 \text { años } \\
\text { Neg. }\end{array}$ \\
\hline 10 & Disgerminoma & I A & Anexohisterect & 3 años Neg. \\
\hline 11 & Disgerminoma & I A & S.O.I. & A $\operatorname{los} 8$ años Neg. \\
\hline 12 & Disgerminoma & IV & $\begin{array}{l}\text { Anexohist. } \\
\text { Radioterapia }\end{array}$ & 3 años $\mathrm{Neg}$. \\
\hline 13 & T.C. Granulosa & III & Anexohist. & Muris al mes. \\
\hline 14 & T.C. Granulosa & I A & S.O.I & 3 años Neg. \\
\hline 15 & $\begin{array}{l}\text { Cistoadenocarcinoma } \\
\text { Seroso. }\end{array}$ & I A & Anexohist. & 3 años $\mathrm{Neg}$. \\
\hline 16 & $\begin{array}{l}\text { Cistoadenocarcinoma } \\
\text { Seroso. }\end{array}$ & I A & (Hc. 317996$)$ & $?$ \\
\hline 17 & $\begin{array}{l}\text { Cistoadenocarcinoma } \\
\text { Mucinoso }\end{array}$ & III & $\begin{array}{l}\text { Anexohist. } \\
\text { Quimioterapia. }\end{array}$ & 17 años $\mathrm{Neg}$. \\
\hline 18 & Adenocarcinoma & III & $\begin{array}{l}\text { Radioterapia } \\
\text { Anexohisterect. }\end{array}$ & 5 años Neq. \\
\hline
\end{tabular}


En la actualidad los resultados son mucho más favorables.

Hay tumores como los de senos endodérmicos que tienen una pobre recuperación del paciente una vez diseminados fuera del ovario, no obstante todas las medidas terapéu ticas aplicadas.

Los tumores del estroma gonadal son poco malignos cuando se presentan en niñas y adolescentes, el tratamiento de- penderá del estadio clínico del tumor y del grado de diferenciación celular.

Los tumores derivados del epitelio celómico son de una alta mortalidad; en esta categoría se considera un método conservador de tratamiento sólo en casos de baja malignidad y estados clínicos tempranos como puede ser los tumores mucinosos, la lesión serosa es tan impredecible en su curso que incluso las lesiones de baja malignidad deben ser tratados como en el adulto ya que su potencial biológico es mucho mayor que en las lesiones mucinosas (5).

\section{TIPO HISTOLOGICO}

Tumor de Senos Endodérmicos

\begin{tabular}{|c|c|c|}
\hline ESTADO C. & TRATA MIENTO & RECUPERACION \\
\hline III & SOI RAD & 5 meses \\
III & $\begin{array}{l}\text { SOD } \\
\text { AHA T. RAD } \\
\text { Quimiot. }\end{array}$ & 13 meses \\
III & Radioterapia & 2 meses \\
\hline
\end{tabular}

TIPO HISTOLOGICO

Disgerminoma

\begin{tabular}{|l|l|c|}
\hline ESTADIO C. & TRATA MIENTO & RECUPERACION \\
\hline I A & SOI & 9 años vive \\
III & AH.A.T. radioterapia & 2 meses \\
III & Radioterapia & 15 meses \\
III & Radioterapia & 1 mes \\
III & A.H.A.T. radioterapia & 5 años viva \\
I A & A.H.A.T. & 3 años vive \\
I A & SOI & 8 años viva \\
IV & A.H.A.T. radioterapia & 3 años viva \\
\hline
\end{tabular}




\section{TIPO HISTOLOGICO}

Tumor de Células de la Granulosa

\begin{tabular}{|l|c|c|c|}
\hline TIPO HISTOLOGICO & ESTADO C. & TRATA MIENTO & RECUPERACION \\
\hline $\begin{array}{l}\text { Tumor de células } \\
\text { de la Granulosa }\end{array}$ & III & A.H.A.T. & 1 mes \\
$\begin{array}{l}\text { Tumor de células } \\
\text { de la Granulosa }\end{array}$ & I A & S O I & 3 años viva \\
\hline
\end{tabular}

TIPO HISTOLOGICO

\begin{tabular}{|l|c|c|c|}
\hline TIPO HISTOLOGICO & Estado C. & Tratamiento & Recuperación \\
\hline $\begin{array}{l}\text { Cistoadenocarcinoma } \\
\text { seroso }\end{array}$ & I A & A.H.A.T. & 3 años viva \\
$\begin{array}{l}\text { Cistoadenocarcinoma } \\
\text { seroso }\end{array}$ & I A & $?$ & $?$ \\
$\begin{array}{l}\text { Cistoadenocarcinoma } \\
\text { Mucinoso }\end{array}$ & III & $\begin{array}{l}\text { AHAT } \\
\text { Quimiotera- } \\
\text { pia }\end{array}$ & 17 años vive \\
\hline
\end{tabular}

TIPO HISTOLOGICO

Adenocarcinoma

\begin{tabular}{|c|l|c|}
\hline ESTADO C. & TRATA MIENTO & RECUPERA CION \\
\hline III & $\begin{array}{l}\text { Radioterapia } \\
\text { AHAT }\end{array}$ & 5 años vive \\
\hline
\end{tabular}




\section{CONCLUSIONES}

Se debe tener en cuenta que la edad no descarta la posibilidad de un tumor maligno del ovario.

Es de anotar que los síntomas que llevaron al diagnóstico del tumor pueden ser obtenidos por su historia clínica y un examen físico adecuado.

En este estudio en el $90 \%$ de los casos el diagnóstico se orientó por la palpación abdominal, a diferencia de estudios en la literatura donde el síntoma cardinal fue cl dolor $(6,7,8,9,10,11,12,13,14)$.

En este estudio al igual que en diferentes publicaciones $(15,16,17)$. Los tu-

\section{BIBLIOGRAFIA}

1. ACOSTA A. KAPLAN A; KAUFMAN R: Gynecologe Cancer in Children. Am. J. Obstet Gynecol 112: $944-1972$.

2. BERGER N., POCHACZEVSKY R.: Astrocytoma - Containing Ovarian Teratoma in Childhood, Am. J. Roetgenol 107: 647, 1969.

3. KILMAN J., WALDHAUSEN J. VELLIOS F., BATTERSBY J. Ovarian Tumors in infants and Children.. Am J. Surg 113: 772, 1967.

4. THOMPSON J. DOCKERTY M., SYMMONDS R. HAYLES A: Ovarian and Paraovarian Tumors in infants and Children. Am. J. Obstet Gynecol 97: 1059, 1967.

5. BREEN J.; L. MAXSON W. S.: Tumores ováricos en niñas y adolescentes.

6. ASADOURIAN La; TAYLOR HB: Dysgerminona, an analysis of 105 cases. Obstet Gynecol 33: 370, 1969. mores de las células germinales fueron los más frecuentes para diversos grupos de edad, y los originados del epitelio celómico aparecen después de la pubertad y aumenta su incidencia con la edad para llegar a ser los más frecuentes en la mujer adulta.

El disgerminoma fue el tumor más frecuente $44 \%$ Siguió en frecuencia el tumor de Senos Endodérmicos 22.2\%.

Aunque la frecuencia de tumores malignos del ovario en niñas y adolescentes es baja, el grado de malignidad es asombroso cuando se compara con los tumores malignos del ovario en la mujer adulta. Esto justifica una búsqueda de esta patología y su manejo adecuado.

7. EINS, DART JMMM, STEPHEN CA.: Cystic and Solid Ovarian Tumors in Children a 44 year review. J. Pediatr Surg 5: 148, 1970.

8. HARRIS, B, BOLES ET: Rational Surgery for Tumors of the Ovary in Children, J. Pediatr Surg 9: 289, 1974.

9. KILMAN J. WALDHAUSEN H.J, VELLIOS F., BATTERSBY J, Ovarian Tumors in infants and Children. Am. J. Surg 113: 772, 1967.

10. MOORE J.G., SCHIFRIN BS., EREZ S.: Ovarian Tumors in infancy, Childhood and Adolescence. Am. J. Obstet Gynecol 99: 913, 1967.

11. NILSON OV., Ovarian Tumors in Children. Acta Obstet Gynecol Scand 47: 119, 1968.

12. STASEK V., VESELY K., MOTLIK $K$.: Malingnant Ovarian Tumors in Children and Adolescents Neoplasma. 18: 299. 1971.

13. THATCHER D.: Ovarian Cysts and Tumors in Children. Surg Gynecol Obstet 117: 477, 1963. 
14. TOWNE B., MAHOUR GH. WOOLEY MM. ISAACS H.: Ovarian Cysts and Tumors in Childhood. J. Pediatr. Surg. 10: 311, 1975.

15. GROEBER W. Ovarian Tumors During infancy and Childhood. AM. J. Obstet Gynecol 86: 1027, 1963.
16. MOORE JG; SCHIFRIN BS;EREZ S: Ovarian Tumors in infancy, Childhood and Adolescent. Am. J. Obstet Gynecol 99: 913, 1967.

17. NORRIS H. JENSEN R.: Relative frequency of Ovarian Neoplasms in Children and Adolescents. Cancer 30: 713, 1972. 